\title{
Quantitative Susceptibility Mapping after Sports-Related Concussion
}

\author{
(D).M. Koch, (D)T.B. Meier, (D) R. Karr, (D)A.S. Nencka, (D).T. Muftuler, and (D) M. McCrea \\ o* $=$
}

\section{ABSTRACT}

BACKGROUND AND PURPOSE: Quantitative susceptibility mapping using MR imaging can assess changes in brain tissue structure and composition. This report presents preliminary results demonstrating changes in tissue magnetic susceptibility after sports-related concussion.

MATERIALS AND METHODS: Longitudinal quantitative susceptibility mapping metrics were produced from imaging data acquired from cohorts of concussed and control football athletes. One hundred thirty-six quantitative susceptibility mapping datasets were analyzed across 3 separate visits (24 hours after injury, 8 days postinjury, and 6 months postinjury). Longitudinal quantitative susceptibility mapping group analyses were performed on stability-thresholded brain tissue compartments and selected subregions. Clinical concussion metrics were also measured longitudinally in both cohorts and compared with the measured quantitative susceptibility mapping.

RESULTS: Statistically significant increases in white matter susceptibility were identified in the concussed athlete group during the acute (24 hour) and subacute (day 8) period. These effects were most prominent at the 8-day visit but recovered and showed no significant difference from controls at the 6-month visit. The subcortical gray matter showed no statistically significant group differences. Observed susceptibility changes after concussion appeared to outlast self-reported clinical recovery metrics at a group level. At an individual subject level, susceptibility increases within the white matter showed statistically significant correlations with return-to-play durations.

CONCLUSIONS: The results of this preliminary investigation suggest that sports-related concussion can induce physiologic changes to brain tissue that can be detected using MR imaging-based magnetic susceptibility estimates. In group analyses, the observed tissue changes appear to persist beyond those detected on clinical outcome assessments and were associated with return-to-play duration after sports-related concussion.

ABBREVIATIONS: $\mathrm{CV}=$ coefficient of variation; $\mathrm{MNI}=$ Montreal Neurological Institute; $\mathrm{mTBI}=$ mild traumatic brain injury; $\mathrm{QSM}=$ quantitative susceptibility mapping; RTP = return to play; SAC = Standardized Assessment of Concussion; SCAT-3 = Sport Concussion Assessment Tool, 3rd ed; SRC = sports-related concussion

M ild traumatic brain injury (mTBI) is an issue of increasing importance in the medical, sports, and military communities. Despite the increasing scope of this issue, the role of diagnos-

Received September 5, 2017; accepted after revision April 8, 2018.

From the Departments of Radiology (K.M.K., R.K., A.S.N.) and Neurosurgery (T.B.M., L.T.M., M.M.), Medical College of Wisconsin, Milwaukee, Wisconsin.

This work was supported, in part, by the GE-NFL Head Health Challenge I and the U.S. Army Medical Research and Materiel Command under award number W81XWH-12-1-0004. This publication was also supported by the Clinical and Translational Science Institute grant 1UL1-RR031973 (-01) and by the National Center for Advancing Translational Sciences, National Institutes of Health grant

8UL1TR000055. It was also supported by the Advancing a Healthier Wisconsin Research and Education Committee.

The contents of this publication are the sole responsibility of the authors and do not necessarily represent the official views of the National Institutes of Health. Opinions, interpretations, conclusions, and recommendations are those of the authors and are not necessarily endorsed by the NFL, GE, or the U.S. Army.

Please address correspondence to Kevin M. Koch, PhD, Medical College of Wisconsin, Department of Radiology, 8701 Watertown Plank Rd, Milwaukee, WI 53226; e-mail: kmkoch@mcw.edu; @KevinMatKoch tic imaging in its assessment and management remains limited. Although CT and MR imaging can detect hemorrhages, contusions, or edema indicative of complicated $\mathrm{mTBI},{ }^{1}$ conventional diagnostic imaging assessments of uncomplicated mTBI have limited sensitivity to the subtle physiologic and morphologic changes in brain function and structure.

MR imaging has recently shown accelerated improvement in performing quantitative assessments of soft-tissue characteristics. As a nonionizing radiative imaging technique, quantitative MR imaging is well-suited for use as a longitudinal scientific probe of the subtle physiologic changes resulting from mTBI. Previous studies have explored mTBI-induced changes in MR imaging dif-

\footnotetext{
-- Indicates open access to non-subscribers at www.ajnr.org

Indicates article with supplemental on-line tables.

http://dx.doi.org/10.3174/ajnr.A5692
} 
fusion tensor imaging, ${ }^{2}$ diffusional kurtosis imaging, ${ }^{3}$ arterial spin-labeling, ${ }^{4}$ and functional connectivity. ${ }^{5}$

Here, we present a preliminary application of another emerging MR imaging quantitative metric, quantitative susceptibility mapping (QSM), in the assessment of mTBI. Conventional susceptibility-weighted imaging, which uses magnetic susceptibility as a contrast mechanism, is well-established as a useful diagnostic tool. ${ }^{6}$ As a quantitative extension of SWI, QSM uses off-resonance information extracted from multiecho MR imaging acquisitions to estimate an isotropic magnetic susceptibility tensor for each tissue voxel. Quantifying the isotropic magnetism of brain tissue can reveal changes in components such as ferritin, hemosiderin, water content, myelin, and calcium. ${ }^{7,8}$ Along with conventional SWI, QSM has already been used to identify regions of focal tissue damage in complicated mTBI in cohorts of military personnel ${ }^{9}$ and civilians. ${ }^{10}$

The present report summarizes preliminary results from QSM MR imaging analysis after sports-related concussion (SRC) in high school and collegiate football athletes. This study is unique compared with previous QSM mTBI studies in that it examines QSM of injured and control subjects longitudinally at 3 visits beginning at the acute injury phase (ie, within 24 hours) and ending 6 months postinjury.

\section{MATERIALS AND METHODS \\ Subjects}

Collegiate and high school football athletes were recruited at preseason team meetings at 10 local institutions. Subjects provided written consent or assent and parental consent if minors for a study approved by the local institutional human research review. A subset of 56 subjects, split evenly across injured and matched control athletes, were enrolled into an advanced MR imaging component of the study during the season. Athletes were imaged within 24 hours after injury, followed by examinations at 8 days and 6 months postinjury. The injured athlete return-to-play (RTP) timeframe was not fixed to the MR imaging session schedule.

One hundred thirty-six QSM datasets collected across the 3 visits were used for the present analysis. Details of longitudinal data composition are provided in On-line Table 1. The Sport Concussion Assessment Tool, 3rd ed (SCAT-3) symptom checklist, the Standardized Assessment of Concussion (SAC), and the Balance Error Scoring System were also collected at each visit.

\section{Imaging and Mapping Technique}

Imaging was performed on a clinical 3T MR imaging scanner using a 32-channel head receive array. QSM data were collected by saving the raw $k$-space data from a commercially available SWI application. SWI data acquisition parameters were as follows: inplane data matrix, $320 \times 256$; FOV , $24 \mathrm{~cm}$; slice thickness, $2 \mathrm{~mm}$; echo spacing, $7 \mathrm{~ms}$; number of echoes, 4; TEs, 10.4, 17.4, 24.4, 31.4 $\mathrm{ms}$; TR, $58.6 \mathrm{~ms}$; autocalibrated parallel imaging factors, $3 \times 1$; acquisition time, 4 minutes.

Following background field removal using the regularizationenabled sophisticated harmonic artifact reduction for phase data

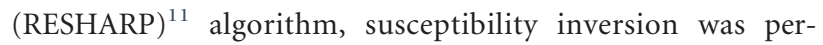
formed using an adapted localized processing formulation ${ }^{12}$ of the morphology-enabled dipole inversion (MEDI) algorithm. ${ }^{13}$
Streaking artifacts are a well-known confounding factor in QSM. ${ }^{7}$ To mitigate these potential artifacts from erroneous field estimates on the periphery of the brain, we applied tissue segmentations and aggressive ( $4 \mathrm{~mm}$ ) erosions to construct a tissue mask. This conservative approach largely eliminated boundary field discontinuities and kept streaking below any qualitatively visible threshold. In addition, a localized processing adaptation of the MEDI algorithm was used to further reduce the streaking impact. ${ }^{12}$ Because of these measures, no datasets were excluded due to streaking artifacts in the manual quality control analysis. Of the 143 acquired QSM datasets, only 7 were removed due to motion-related quality control checks.

\section{Image Analysis}

Group analyses were performed via a triple-stage registration process implemented in FSL (http://www.fmrib.ox.ac.uk/fsl). ${ }^{14}$ First, magnitude images from a 3D T1-weighted scan collected on each subject were nonlinearly registered to a $2-\mathrm{mm}$ isotropic Montreal Neurological Institute (MNI) space T1-weighted template image. ${ }^{15}$ Next, a magnitude image constructed from each subject's QSM acquisition was affine-registered to the subject's T1-weighted image. All QSMs were then transformed to MNI space and registered to a single common QSM dataset in MNI space using FSL. ${ }^{15}$

Group changes in susceptibility were identified through the following processing pipeline: First, brain regions of sufficient QSM stability were identified by computing coefficients of variation (CVs) within the control cohort. A stability mask was then constructed from this CV map at a threshold of CV $<0.8$. Next, global white matter and iron-rich subcortical gray matter compartments were defined using susceptibility thresholds computed on a mean susceptibility map of the control subjects. White matter was defined as $\chi<-0.03 \mathrm{ppm},{ }^{16}$ and subcortical iron-rich regions were defined as $\chi>0.05 \mathrm{ppm}$.

ROI analysis within the compartments was performed using anatomic segmentations extracted from the Johns Hopkins University and Harvard MNI space atlases. ${ }^{17,18}$ Primary analyses focused on 2 global compartments (ie, white matter and subcortical gray matter). Twenty-eight additional ROIs within the global compartments were assessed as secondary analyses. These regions are summarized in Table 1.

\section{Statistical Analysis}

Statistical processing was performed using the Statistics Toolbox in Matlab (MathWorks, Natick, Massachusetts). Mean susceptibility values were computed within each gray and white matter ROI for each subject. Group differences between the means were computed using 2-tailed independent samples $t$ tests with unequal variances at each visit. In addition, effect sizes between the groups were estimated by computing the Cohen $\mathrm{D}$ at each visit. Longitudinal trends of the susceptibility means were assessed using a linear mixed effects model with random effects terms. In the linear mixed effects model, the mean ROI susceptibility was the response variable and the direct fixed predictor terms were group (injured versus control) and visit. An interaction fixed predictor term, group $\times$ visit, was also included in the model. Finally, random effects terms were modeled for subject variation by group and subject variation by visit. Pearson correlations between sus- 
ceptibility measurements and clinical symptoms (SCAT-3, SAC, Balance Error Scoring System) were performed for each region at each visit. RTP outcome measures were also correlated with the 24-hour susceptibility measurements. For the subregional analyses, multiple comparison corrections were performed using the Benjamini-Hochberg false discovery rate correction at a false discovery rate of $10 \%$.

\section{RESULTS}

Tables 2 and 3 provide general characteristics of the complete study population. None of the reported physical or general

Table 1: Description of additional ROls in white matter and subcortical gray matter

\begin{tabular}{ll}
\hline \multicolumn{1}{c}{ White Matter ROI } & Gray Matter ROI \\
\hline L anterior thalamic radiation & L thalamus \\
R anterior thalamic radiation & L caudate \\
L corticospinal tract & L putamen \\
R corticospinal tract & L pallidum \\
L cingulum (cingulate gyrus) & R thalamus \\
R cingulum (cingulate gyrus) & R caudate \\
L cingulum (hippocampus) & R putamen \\
R cingulum (hippocampus) & R pallidum \\
Forceps major & \\
Forceps minor & \\
L inferior fronto-occipital fasciculus & \\
R inferior fronto-occipital fasciculus & \\
L inferior longitudinal fasciculus & \\
R inferior longitudinal fasciculus & \\
L superior longitudinal fasciculus & \\
R superior longitudinal fasciculus & \\
L uncinate fasciculus & \\
R uncinate fasciculus & \\
L superior longitudinal fasciculus (temporal) & \\
R superior longitudinal fasciculus (temporal) & \\
\hline
\end{tabular}

Note:- R indicates right; L, left. metrics showed statistically significant group differences, though the history of prior diagnosed sports-related concussions between the groups nearly reached significance $(P=.07$, Mann-Whitney $U$ test). SCAT-3, SAC, Balance Error Scoring System, and RTP measures on the full study cohort are also presented in Tables 2 and 3. A breakdown of the cohort characteristics based on QSM data inclusion at each visit is provided in On-line Table 2.

Figure 1 presents control group susceptibility mean $(A), \mathrm{SD}$ $(B)$, and coefficient of variation $(C)$ maps across several axial slices. The CV maps provide an estimate of QSM stability, as measured across the control group. This stability assessment reflects physiologic, acquisition, and algorithmic variations. The susceptibility estimate is reliable in particular brain regions comprising deep gray matter structures and deep white matter tracts.

Figure $1 D$ shows subcortical gray matter (blue) and white matter (red) regions that were identified on MNI atlases and masked to reflect regions of sufficient susceptibility measurement stability $(\mathrm{CV}<0.8)$. Table 1 provides descriptions for each of the assessed ROIs.

Table 4 summarizes the group susceptibility effects for several regions, including the global white and gray matter compartments. The global white matter compartment showed statistically significant susceptibility increases relative to the control group at both the 24-hour and 8-day visits. This effect was diminished and was no longer statistically significant at the 6-month visit. Several displayed individual white matter regions showed similar trends. Eleven of the 20 white matter subregions showed a statistically significant positive increase at 1 or both of the 24-hour and/or 8 -day visits. One subregion (the right cingulum) showed significant group differences remaining at the 6-month visit. Adjustment of these results by at $10 \%$ false discovery rate correction

Table 2: General characteristics of complete control and injured study cohorts $(N=56)^{a}$

\begin{tabular}{|c|c|c|c|c|c|c|c|}
\hline & \multicolumn{3}{|c|}{ Physical } & \multicolumn{4}{|c|}{ General } \\
\hline & $\begin{array}{c}\text { Age } \\
(n=28)(y r)\end{array}$ & $\begin{array}{c}\text { Weight } \\
(n=28) \text { (lbs) }\end{array}$ & $\begin{array}{c}\text { Height } \\
(n=28) \text { (in) }\end{array}$ & $\begin{array}{c}\text { Y.I.S. } \\
(n=28)(y r)\end{array}$ & $\begin{array}{c}\text { G.P.A. } \\
(n=28)(4.0)\end{array}$ & $\begin{array}{c}\text { P.SRC }(n=28) \\
\text { (Total) }\end{array}$ & $\begin{array}{c}\text { RTP } \\
(n=24)(\text { days })\end{array}$ \\
\hline C & 17.9 (17.3-18.5) & 202 (188-217) & $72.0(71.0-73.0)$ & $8.3(7.2-9.5)$ & $3.3(3.1-3.5)$ & 8 & NA \\
\hline I & $17.7(17.2-18.3)$ & $221(199-243)$ & 71.6 (70.4-72.8) & $8.6(7.3-9.8)$ & $3.1(2.8-3.3)$ & 16 & $\begin{array}{c}13.9 \text { (11.3-16.5) } \\
\text { range, 4-28 }\end{array}$ \\
\hline$P$ & .66 & .15 & .64 & .76 & .11 & .07 & NA \\
\hline
\end{tabular}

Note:-Y.I.S. indicates years in sport; G.P.A., academic grade point average on a 4.0-point scale; P.SRC, number of previous sports-related concussions; C, control; I, injured; NA, not applicable.

${ }^{a}$ When appropriate, values are shown as mean and $95 \%$ confidence intervals of the distribution. $P$ values from 2-tailed independent samples between the 2 cohorts are reported for all metrics. Due to skewness of the distribution, the P.SRC test statistics were computed using a Mann-Whitney U test. Return to play: 3 of the 24 injured subjects returned to play prior to the 8-day imaging visit.

Table 3: Clinical characteristics of complete control and injured study cohorts $(N=56)^{a}$

\begin{tabular}{|c|c|c|c|c|c|c|c|c|c|}
\hline & \multicolumn{3}{|c|}{ SCAT Severity } & \multicolumn{3}{|c|}{ SAC Total Score } & \multicolumn{3}{|c|}{ BESS Score } \\
\hline & 24 Hours & 8 Days & 6 Months & 24 Hours & 8 Days & 6 Months & 24 Hours & 8 Days & 6 Months \\
\hline \multirow[t]{3}{*}{$C$} & $(n=28)$ & $(n=28)$ & $(n=19)$ & $(n=28)$ & $(n=28)$ & $(n=22)$ & $(n=27)$ & $(n=27)$ & $(n=22)$ \\
\hline & 3.2 & 2.9 & 2.1 & 26.1 & 27.6 & 27.2 & 12.3 & 10.8 & 11.4 \\
\hline & $(1.9-4.5)$ & $(1.2-4.6)$ & $(0.8-3.4)$ & $(25.4-26.8)$ & $(27.0-28.2)$ & $(26.4-28.0)$ & $(10.5-14.0)$ & $(8.8-12.8)$ & (9.5-13.2) \\
\hline \multirow[t]{3}{*}{ I } & $(n=28)$ & $(n=28)$ & $(n=19)$ & $(n=28)$ & $(n=28)$ & $(n=17)$ & $(n=25)$ & $(n=28)$ & $(n=18)$ \\
\hline & 27.8 & 5.0 & 3.1 & 24.6 & 26.8 & 26.9 & 28.9 & 5.5 & 1.2 \\
\hline & $(21.4-34.2)$ & $(1.9-8.2)$ & $(-1.1-7.4)$ & $(23.7-25.5)$ & $(25.9-27.7)$ & $(25.8-27.9)$ & (11.8-15.7) & (10.2-13.7) & (9.9-14.4) \\
\hline$P$ & $<.001^{b}$ & .22 & .64 & $.01^{\mathrm{b}}$ & .14 & .63 & .25 & .36 & .57 \\
\hline
\end{tabular}

Note:-BESS indicates Balance Error Scoring System; C, control; I, injured.

${ }^{a}$ When appropriate, values are shown as mean and $95 \%$ confidence intervals of the distribution. $P$ values from 2 -tailed independent samples between the 2 cohorts are reported for all metrics.

${ }^{\mathrm{b}}$ Significant. 
yielded 10 statistically significant subregions at the 8-day visit. None of the $t$ tests of the control visit groups relative to the full control group showed any significant differences (before any false discovery rate correction). Although there were not any

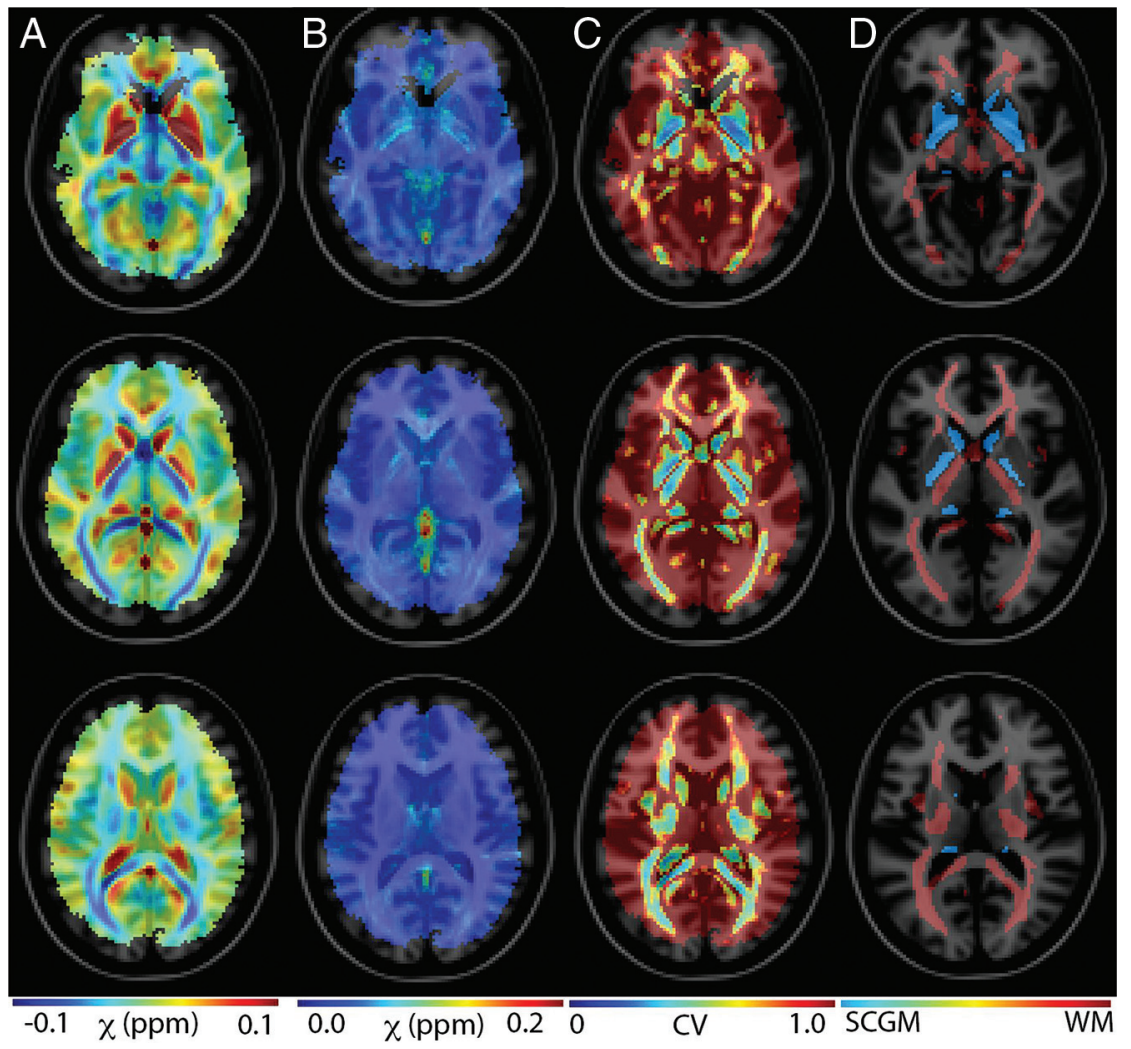

FIG 1. Assessment of QSM stability across the control group. Maps of mean (A), SD (B), and coefficients of variation $(C)$ are shown across 4 axial slices throughout the brain. The stability of the QSM measurement varies across brain compartments. Deep gray and white matter structures show the most prominent stability. D, Subcortical gray matter (blue) and white matter (red) regions that were identified on $\mathrm{MNI}$ atlases and masked to reflect regions of sufficient susceptibility measurement stability $(\mathrm{CV}<0.8)$. statistically significant effects in the subcortical gray matter compartments, the measured subcortical susceptibility differences generally showed opposing effect signs compared with the white matter.

Figure 2 presents boxplots of 4 representative white matter regions. The global white matter compartment is shown in $A$. A general upward trend in susceptibility values is observed in the injured group, which has statistical significance relative to the controls at the 24-hour and 8-day visits. This difference is removed by the 6-month visit. Plots $B$, $C$, and $D$, respectively, provide similar analysis within 2 longitudinal fasciculus regions and 1 thalamic radiation subregion. Similar longitudinal trends are observed in these areas.

Neither of the global or subregional tissue compartments showed statistically significant correlations between the acute (24 hour) visit susceptibility and the SCAT-3, SAC, or Balance Error Scoring System metrics within the injured group. However, there was a significant positive correlation (after false discovery rate correction) between the injured acute susceptibility measurements and RTP in several white matter compartments that showed significant group susceptibility differences (Table 4).

Linear mixed effects model results are shown in Table 5. The global white matter and several subregions (2 re-

Table 4: Statistical report comparing means of susceptibility values within injured and control groups ${ }^{\mathrm{a}}$

\begin{tabular}{|c|c|c|c|c|c|c|c|c|}
\hline \multirow[b]{3}{*}{ ROI } & \multicolumn{6}{|c|}{ Susceptibility Group Differences (Injured vs Control) } & \multirow{2}{*}{\multicolumn{2}{|c|}{$\begin{array}{c}\text { RTP vs } \\
\text { 24-Hour Suscept }\end{array}$}} \\
\hline & \multicolumn{2}{|c|}{24 Hours } & \multicolumn{2}{|c|}{8 Days } & \multicolumn{2}{|c|}{6 Months } & & \\
\hline & $P$ & D & $P$ & D & $P$ & D & $P$ & $\boldsymbol{\rho}$ \\
\hline White matter & $.032^{\mathrm{b}}$ & $0.65^{\mathrm{b}}$ & $.001^{\mathrm{b}}$ & $1.01^{\mathrm{b}}$ & .463 & 0.25 & $.022^{\mathrm{b}}$ & 0.55 \\
\hline Gray matter & .980 & -0.01 & .255 & -0.34 & .382 & -0.30 & .146 & -0.26 \\
\hline L ATR & $.017^{\mathrm{b}}$ & $0.72^{b}$ & .069 & 0.55 & .735 & -0.11 & .146 & 0.37 \\
\hline R cing (hipp) & .072 & 0.53 & $.011^{b, c}$ & $0.79^{b}$ & $.012^{\mathrm{b}}$ & $0.91^{\mathrm{b}}$ & $.015^{\mathrm{b}, \mathrm{c}}$ & $0.58^{b}$ \\
\hline Forceps minor & $.017^{\mathrm{b}}$ & $0.75^{b}$ & $.008^{b, c}$ & $0.82^{b}$ & .738 & 0.11 & .989 & $<0.01$ \\
\hline L IFOF & .127 & 0.46 & $.037^{\mathrm{b}, \mathrm{c}}$ & $0.63^{b}$ & .730 & 0.12 & $.015^{\mathrm{b}, \mathrm{c}}$ & $0.58^{b}$ \\
\hline R IFOF & .073 & 0.55 & $.019^{\mathrm{b}, \mathrm{c}}$ & $0.72^{b}$ & .783 & 0.09 & .480 & 0.18 \\
\hline L ILF & .194 & 0.39 & $.027^{\mathrm{b}, \mathrm{c}}$ & $0.67^{\mathrm{b}}$ & .650 & 0.15 & .146 & 0.37 \\
\hline R ILF & .187 & 0.40 & $.034^{\mathrm{b}, \mathrm{c}}$ & $0.64^{b}$ & .814 & 0.08 & $.003^{b, c}$ & $0.67^{b}$ \\
\hline L SLF & .334 & 0.28 & $.014^{\mathrm{b}, \mathrm{c}}$ & $0.75^{b}$ & .550 & 0.21 & $.019^{\mathrm{b}, \mathrm{c}}$ & $0.56^{b}$ \\
\hline R SLF & $.021^{\mathrm{b}}$ & $0.72^{\mathrm{b}}$ & $.002^{\mathrm{b}, \mathrm{c}}$ & $1.00^{\mathrm{b}}$ & .819 & 0.08 & $.015^{\mathrm{b}, \mathrm{c}}$ & $0.58^{b}$ \\
\hline L SLF (temp) & .847 & 0.06 & $.035^{b, c}$ & $0.64^{b}$ & .510 & 0.23 & $.034^{\mathrm{b}, \mathrm{c}}$ & $0.52^{b}$ \\
\hline R SLF (temp) & $.010^{\mathrm{b}}$ & $0.82^{b}$ & $.007^{b, c}$ & $0.83^{b}$ & .576 & 0.19 & .412 & 0.21 \\
\hline
\end{tabular}

Note:-ATR indicates anterior thalamic radiation; cing, cingulum; hipp, hippocampus; IFOF, inferior fronto-occipital fasciculus; ILF, inferior longitudinal fasciculus; SLF, superior longitudinal fasciculus; temp, temporal; RTP, return to play temporal duration; D, effect sizes; Suscept, susceptibility; L, left; R, right.

a White matter regions showing significant trends and the global gray matter compartment are shown. At each visit, group differences are characterized with $P$ values computed using 2-tailed independent samples $t$ tests and effect sizes are computed using the Cohen D. The Pearson correlation of RTP temporal duration vs 24 -hour susceptibility values for individual injured subjects is also presented. $P$ values and effect sizes $(\rho)$ are displayed. $P$ values for the subregional analyses are reported without multiple-comparison corrections.

${ }^{b}$ Effects that showed statistical significance $(P<.05)$.

'Statistically significant after correction for multiple comparisons. 

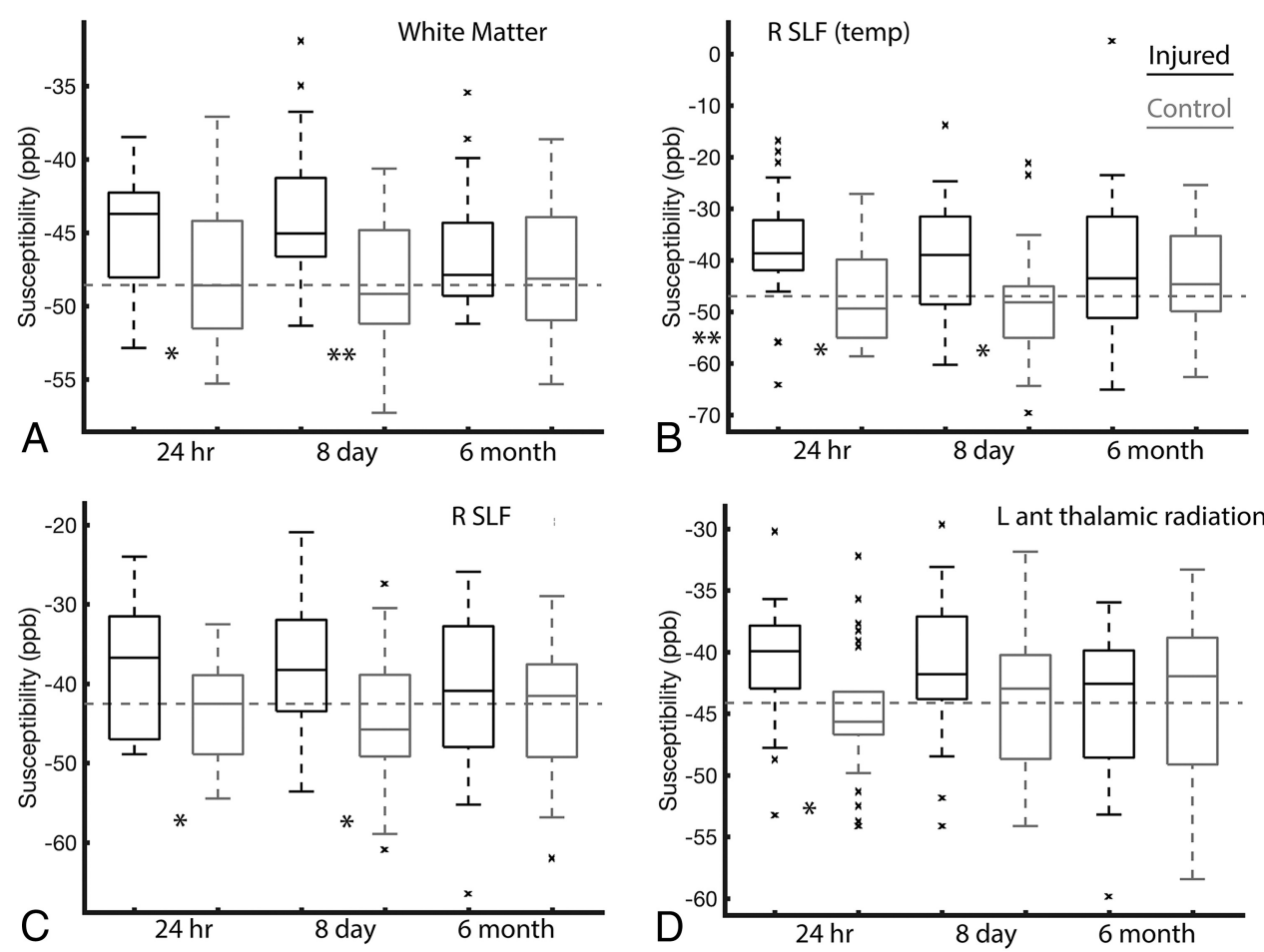

FIG 2. Boxplots of susceptibility means across 4 representative white matter regions. Indices for each region, as defined in Table 1, are provided for each boxplot. The displayed boxplots provide median lines, boxes across the interquartile range, and bars across the 95th confidence interval regions. Outliers are plotted as $x$ 's. The dashed horizontal line provides a visual of the full control cohort mean susceptibility value. Statistical significance of the injured-versus-control $t$ tests at each visit are indicated for $P<.05$ (asterisk) and $P<.01$ (double asterisks). SLF indicates superior longitudinal fasciculus; ant, anterior; temp, temporal; L, left; R, right.

Table 5: Linear mixed effects modeling results ${ }^{a}$

\begin{tabular}{|c|c|c|c|}
\hline \multirow[b]{2}{*}{ ROls } & \multicolumn{2}{|c|}{ Direct } & \multirow{2}{*}{$\frac{\text { Interact }}{\text { Time } \times \text { Injury }}$} \\
\hline & Time & Injury & \\
\hline \multicolumn{4}{|l|}{ Primary } \\
\hline White matter & .874 & $.016^{\mathrm{b}}$ & .659 \\
\hline Gray matter & .390 & .711 & .175 \\
\hline \multicolumn{4}{|l|}{ Additional } \\
\hline L ant. thal. rad. & .416 & $.003^{b, c}$ & .053 \\
\hline Forceps minor & .767 & $.004^{b, c}$ & .256 \\
\hline L IFOF & .986 & $.043^{b}$ & .226 \\
\hline R SLF & .903 & $.023^{b}$ & .671 \\
\hline R SLF (temp) & .498 & $.024^{\mathrm{b}}$ & .500 \\
\hline
\end{tabular}

Note:- ant. thal. rad. indicates anterior thalamic radiation; L, left; R, right; IFOF, inferior fronto-occipital fasciculus; SLF, superior longitudinal fasciculus; temp, temporal. a $P$ values are reported for white matter and gray matter compartments, as well as for additional ROIs with significant effects. Interaction terms indicate longitudinal susceptibility variations of one group relative to the other.

b Statistical significance $(P<.05)$.

c Significant after multiple-comparison correction.

gions, post-false discovery rate correction) showed statistically significant effects of the injured group relative to the controls. None of the regions showed statistically significant interaction terms, which would have indicated statistical recovery patterns within the measurement visits.

\section{DISCUSSION}

Because QSM is very sensitive to blood products, it can clearly identify local damage in complicated mTBI, whereby shearing forces can cause diffuse axonal injury patterns that manifest as hemorrhages and contusions. ${ }^{9}$ The potential utility of QSM in assessing uncomplicated mTBI is more nuanced. In a recent study by Lin et al, ${ }^{10}$ a cohort of clinical subjects admitted for hospital care with mTBI were analyzed with QSM within 2 weeks of injury. In agreement with the results of the present study, Lin et al found susceptibility increases associated with injury in white matter regions. In addition, Lin et al found substantial decreases of susceptibility in subcortical gray matter compartments. Although the present study did not find statistically significant differences in these regions, the general gray matter trends (Table 4) show a negative correlation between susceptibility and injury, thus adding support to recent QSM studies that have found that susceptibility decreases in deep gray matter regions correlated with complicated mTBI. ${ }^{19}$

The magnitude of group susceptibility differences observed in this study is nearly an order of magnitude less than that reported by Lin et al. ${ }^{10}$ This finding is expected, given the reduced magnitude of head trauma in the present sports concussion cohort and could be one reason that the observed subcortical gray matter susceptibility reduction trends did not reach statistical significance. In addition, compared with deep gray matter structures, white matter structures may be more sensitive to shear strain forces experienced during mTBI and therefore exhibit detectable differences at a lower injury threshold.

The stability analysis shown in Fig 1 illustrates the practical limitations of QSM in reliably detecting small changes in tissue susceptibility. This expected result shows that QSM estimates will predominantly be most reliable in deep gray and white matter structures. Outside these regions, the control susceptibility estimates are highly variable. This result is not surprising because QSM is known to be dependent on the white matter in tract ori- 
entation with respect to the main MR imaging magnetic field direction. $^{20}$

The physiologic and physical causes of the observed susceptibility changes will require further scientific investigation. Increases in white matter susceptibility could be due to demyelination or increased water content (swelling). Qualitative correlation of QSM and diffusion tensor imaging results from the athlete cohort analyzed in this study ${ }^{3}$ has shown localized agreement in white matter regions of QSM increases and decreased axial diffusion, which support the white matter swelling hypothesis. Preliminary studies suggest that the observed gray matter decreases could be due to calcium (which is a diamagnetic [negative susceptibility] ion) influx after brain trauma. ${ }^{21}$

In recent years, there has been increased attention paid to the assessment and management of SRC. ${ }^{22-28}$ Although large-cohort studies have already informed SRC best practices, ${ }^{29,30}$ management of its progression remains a substantial challenge in the sports medicine community. Several studies ${ }^{29,31-33}$ have provided insight into elements of SRC progression but did not uncover an objective biomarker that relates the acute physiologic effects of SRC on brain structure and function. Without such a biomarker, it is difficult to identify the windows of cerebral vulnerability that may extend beyond the point of clinical recovery. ${ }^{34}$ This period of vulnerability is of major translational significance because it may point to a physiologically compromised brain state, which potentially increases the risk of repetitive injury. As shown in Tables 2 and 3, the group SCAT-3 differences in this study are resolved by the 8-day postinjury assessment. The global white matter compartment and several subregions, however, still show substantial statistically significant group differences at this assessment point. Linear mixed effects models, which accounted for individual subject variations and random effects, showed substantial group susceptibility differences correlating with injury.

The present study had several limitations. First, as with many longitudinal studies of nonclinical cohorts, subject compliance for the MR imaging examination component of the study was not perfect (On-line Table 1) and showed attrition at the last visit point. A necessary mathematic assumption applied to the statistical analysis in this study is that the missing data are randomly distributed between the analysis groups. However, this data attrition could partially explain the lack of a significant interaction term in the linear mixed effects model.

A second limitation of the study is that baseline imaging measurements were not performed. This lack of data acquisition was due to the substantial additional resources that would be required to perform an MR imaging examination on every enrolled subject in the study, $>3000$ athletes. To address this limitation, future studies may target baseline imaging in smaller cohorts that are more likely to experience injury. Although no injured subjects experienced a second diagnosed concussion during the context of the study, injured subjects were exposed to subconcussive impacts throughout the duration of the study after their return to normal activity. In addition, control subjects in this study were also contact sport athletes experiencing subconcussive impacts throughout their participation in the study. Thus, this study was not wellpositioned to study the effects of subconcussive events on tissuesusceptibility measurements.
The lack of baseline imaging highlights another potential confounding factor in the study: the near statistically significant $(P=$ $.07)$ difference in prior SRC history between the injured and control groups. Although this study excluded subjects who had ongoing neurocognitive effects from past brain injuries, injured subjects were not excluded solely on the basis of prior concussions. Control subjects were excluded if they had a diagnosed prior concussion within 6 months of their initial imaging session. To further study the effects of prior concussion on the results of this study, Pearson correlations between the first time point susceptibility values and prior SRC history were computed for both cohort groups. Neither the global white matter region nor any of the subregions with statistically significant group differences showed a statistically significant correlation in these tests. This finding suggests that the history of prior concussion is not a strong determinant of the observed susceptibility changes.

Finally, this study did not analyze highly localized variations of tissue susceptibility within individual subjects. Such effects will not be captured in the ROI analyses used. Future work will apply localized extremum analyses to identify patterns of susceptibility changes within individual subjects.

\section{CONCLUSIONS}

The presented analysis of QSM MR imaging group differences in SRC has shown encouraging preliminary results. Consistent susceptibility differences have been identified in the injured cohort. The directionality of the observed susceptibility changes is in general agreement with larger military and civilian studies of mTBI using QSM, including those studies of more severe TBI. Furthermore, the identified group changes in susceptibility appear to outlast symptom-recovery patterns and correlate with RTP time after SRC. Further work will be required to assess the effect of head impact exposure on the observed tissue-susceptibility recovery patterns.

Disclosures: Timothy B. Meier-RELATED: Grant: GE Head-Health Initiative, Comments: Work was completed as part of the GE-NFL Head Health Challenge I award (Principal Investigator, Mike McCrea).* Andrew S. Nencka-RELATED: Grant: GE Healthcare, Comments: salary support; Principal Investigator, Mike McCrea*; UNRELATED: Grants/Grants Pending: GE Healthcare*; Patents (Planned, Pending or Issued): Medical College of Wisconsin, Comments: The author is listed as an inventor on MRI-related patents owned by Medical College of Wisconsin.* Michael McCrea-RELATED: Grant: National Collegiate Athletic Association and Department of Defense.* *Money paid to the institution.

\section{REFERENCES}

1. Williams DH, Levin HS, Eisenberg HM. Mild head injury classification. Neurosurgery 1990;27:422-28 CrossRef Medline

2. Shenton ME, Hamoda HM, Schneiderman JS, et al. A review of magnetic resonance imaging and diffusion tensor imaging findings in mild traumatic brain injury. Brain Imaging Behav 2012;6:137-92 CrossRef Medline

3. Lancaster MA, Olson DV, McCrea MA, et al. Acute white matter changes following sport-related concussion: a serial diffusion tensor and diffusion kurtosis tensor imaging study. Hum Brain Mapping 2016;37:3821-34 CrossRef Medline

4. Wang Y, Nelson LD, LaRoche AA, et al. Cerebral blood flow alterations in acute sport-related concussion. J Neurotrauma 2016;33: 1227-36 CrossRef Medline

5. Mayer AR, Bellgowan PS, Hanlon FM. Functional magnetic resonance imaging of mild traumatic brain injury. Neurosci Biobehav Rev 2015;49:8-18 CrossRef Medline 
6. Mittal S, Wu Z, Neelavalli J, et al. Susceptibility-weighted imaging: technical aspects and clinical applications, part 2. AJNR Am J Neuroradiol 2009;30:232-52 CrossRef Medline

7. Wang Y, Liu T. Quantitative susceptibility mapping (QSM): decoding MRI data for a tissue magnetic biomarker. Magn Reson Med 2015;73:82-101 CrossRef Medline

8. Reichenbach JR, Schweser F, Serres B, et al. Quantitative susceptibility mapping: concepts and applications. Clin Neuroradiol 2015; 25(Suppl 2):225-30 CrossRef Medline

9. Liu W, Soderlund K, Senseney JS, et al. Imaging cerebral microhemorrhages in military service members with chronic traumatic brain injury. Radiology 2016;278:536-45 CrossRef Medline

10. Lin HH, Liu HS, Tsai PH, et al. Quantitative susceptibility mapping in mild traumatic brain injury. In: Proceedings of the Annual Meeting and Exhibition of the International Society for Magnetic Resonance in Medicine, Honolulu, Hawaii. April 22-27, 2017:2395

11. Sun H, Wilman AH. Background field removal using spherical mean value filtering and Tikhonov regularization. Magn Reson Med 2014;71:1151-57 CrossRef Medline

12. Anderson C, Nencka A, Muftuler T, et al. Volume-parcellated quantitative susceptibility mapping. In: Proceedings of the Annual Meeting and Exhibition of the International Society for Magnetic Resonance in Medicine, Singapore. May 7-13, 2016:1108

13. Liu J, Liu T, de Rochefort L, et al. Morphology enabled dipole inversion for quantitative susceptibility mapping using structural consistency between the magnitude image and the susceptibility map. Neuroimage 2012;59:2560-68 CrossRef Medline

14. Jenkinson M, Beckmann CF, Behrens TE, et al. FSL. Neuroimage 2012;62:782-90 CrossRef Medline

15. Mazziotta J, Toga A, Evans A, et al. A four-dimensional probabilistic atlas of the human brain. J Am Med Inform Assoc 2001;8:401-30 CrossRef Medline

16. Li W, Wu B, Batrachenko A, et al. Differential developmental trajectories of magnetic susceptibility in human brain gray and white matter over the lifespan. Hum Brain Mapp 2014;35:2698-713 CrossRef Medline

17. Desikan RS, Ségonne, Fischl B, et al. An automated labeling system for subdividing the human cerebral cortex on MRI scans into gyral based regions of interest. Neuroimage 2006;31:968-80 CrossRef Medline

18. Mori S, Wakana S, van Zijl PC, et al. MRI Atlas of Human White Matter. Boston: Elsevier; 2005

19. Liu W, Riedy G, Yeh PH, et al. Patients with cerebral microhemorrhage exhibit increased magnetic susceptibility in the cerebral hemispheres, but reduced magnetic susceptibility in the basal ganglia. In: Proceedings of the Annual Meeting and Exhibition of the International Society for Magnetic Resonance in Medicine, Honolulu, Hawaii. April 22-27, 2017:4534

20. Li Wei, Liu C. Comparison of magnetic susceptibility tensor and diffusion tensor of the brain. J Neurosci Neuroeng 2013;2:431-40 CrossRef Medline
21. Schweser F. 9.4 Tesla in vivo quantitative susceptibility mapping (QSM) detects thalamic calcium influx associated with repeated mild traumatic brain injury (mTBI). In: Proceedings of the Annual Meeting and Exhibition of the International Society for Magnetic Resonance in Medicine, Honolulu, Hawaii. April 22-27, 2017:4517

22. McCrory P, Meeuwisse WH, Aubry M, et al. Consensus statement on concussion in sport: the 4th International Conference on Concussion in Sport held in Zurich, November 2012. Br J Sports Med 2013; 47:250-58 CrossRef Medline

23. McCrory P, Meeuwisse W, Johnston K, et al. Consensus statement on concussion in sport: the 3rd International Conference on Concussion in Sport held in Zurich, November 2008. J Athl Train 2009; 44:434-48 CrossRef Medline

24. McCrory P, Johnston K, Meeuwisse W, et al. Summary and agreement statement of the 2nd International Conference on Concussion in Sport, Prague 2004. Br J Sports Med 2005;39:196-204 Medline

25. DeKosky ST, Ikonomovic MD, Gandy S. Traumatic brain injury: football, warfare, and long-term effects. N Engl J Med 2010;363: 1293-96 CrossRef Medline

26. Kelly JP. Traumatic brain injury and concussion in sports. JAMA 1999;282:989-91 CrossRef Medline

27. Langlois JA, Rutland-Brown W, Wald MM. The epidemiology and impact of traumatic brain injury: a brief overview. J Head Trauma Rehabil 2006;21:375-78 CrossRef Medline

28. Centers for Disease Control and Prevention (CDC). Nonfatal traumatic brain injuries from sports and recreation activities: United States, 2001-2005. MMWR Morb Mortal Wkly Rep 2007;56:733-37 Medline

29. McCrea M, Guskiewicz KM, Marshall SW, et al. Acute effects and recovery time following concussion in collegiate football players: the NCAA Concussion Study. JAMA 2003;290:2556-63 CrossRef Medline

30. Guskiewicz KM, McCrea M, Marshall SW, et al. Cumulative effects associated with recurrent concussion in collegiate football players: the NCAA Concussion Study. JAMA 2003;290:2549-55 CrossRef Medline

31. McCrea M, Barr WB, Guskiewicz K, et al. Standard regression-based methods for measuring recovery after sport-related concussion. J Int Neuropsychol Soc 2005;11:58-69 Medline

32. McCrea M, Guskiewicz K, Randolph C, et al. Effects of a symptomfree waiting period on clinical outcome and risk of reinjury after sport-related concussion. Neurosurgery 2009;65:876-82; discussion 882-83 CrossRef Medline

33. McCrea M, Prichep L, Powell MR, et al. Acute effects and recovery after sport-related concussion: a neurocognitive and quantitative brain electrical activity study. J Head Trauma Rehabil 2010;25: 283-92 CrossRef Medline

34. McCrory P, Meeuwisse W, Dvořák J, et al. Consensus statement on concussion in sport: the 5th International Conference on Concussion in Sport held in Berlin, October 2016. Br J Sports Med 2017;51: 838-47 CrossRef Medline 